\title{
HALL-HIGMAN TYPE THEOREMS. IV
}

\author{
T. R. BERGER ${ }^{1}$
}

ABstract. Hall and Higman's Theorem B is proved by constructing the representation in the group algebra. This proof is independent of the field characteristic, except in one case.

Let $R$ be an extra special $r$ group. Suppose $C \leqq \operatorname{Aut}(R)$ is cyclic, irreducible faithful on $R / Z(R)$, and trivial on $Z(R)$. The group $C R$ and its representation theory have been widely studied and are of some importance. Let $\boldsymbol{k}$ be a field of characteristic $q \neq r$ containing a splitting field for $R$. Then any faithful irreducible $k[R]$-module $V$ is absolutely irreducible and extends to $C R$.

Hall and Higman [3] studied $\left.V\right|_{C}$ when $C$ was a $q$ group. Their result is proved using inequalities on the dimension of $\operatorname{Hom}_{k[C]}(V, V)$. This proof also works if we only know $q|| C \mid$. The central ideal of this proof also works when $(q,|C|)=1$ [5]. However, the count is quite different. There are character proofs of the result when $(q,|C|)=1[1]$. Thompson gave a very pretty proof of Hall and Higman's original result using vertices and sources [4].

These proofs suffer from one or more of the following difficulties:

(1) They are all tied to the characteristic $q$.

(2) When $q|| C \mid$ they depend upon knowing all indecomposable $k[C]$ modules.

(3) They are indirect in that they do not construct a representation of $C R$.

The theorem in all cases is that $\left.V\right|_{C}$ is a direct sum of copies of the regular $C$-module and one other module $U$ isomorphic to $k[C] / k[C] F$ where $F=$ $\sum_{x \in C} x$. The number of regular modules and the appearance of $U$ are completely independent of the field characteristic. This makes (1) a strong objection. Objection (2) becomes important if we drop the condition that $C$ be cyclic. Finally, a "useful" construction of a given module is often better than no construction at all.

Received by the editors February 11, 1972.

AMS (MOS) subject classifications (1970). Primary 20C15, 20C20; Secondary 16A64, $20 \mathrm{H} 20$.

Key words and phrases. Hall-Higman Theorem B, representation theory, group theory, group algebra, modular representation.

${ }^{1}$ This research was partially supported by NSF grant GP-29224X.

(c) American Mathematical Society 1973 
The object of this paper is to remedy these three objections for odd primes $r$. The representation of $C R$ on $V$ is explicitly constructed independent of field characteristic. Then its restriction to $C$ is studied. If $|R|=r^{2 e+1}$ then it is shown that $\left.V\right|_{C}$ contains $\left(r^{e}+1\right) /|C|-1$ copies of the regular $C$ module and one copy of $k[C] / k[C] F$.

\section{A remark on central simple algebras.}

(1.1) Suppose $\boldsymbol{k}$ is a field and $A$ is a central simple algebra over $\boldsymbol{k}$ of dimension $t^{2}$. Assume $B$ is a commutative quasi-Frobenius subalgebra with 1. Let $V$ be an irreducible $A$-module. Then $\left.V\right|_{B} \simeq{ }_{B} B+U$ where ${ }_{B} B$ is $B^{+}$considered as a left $B$-module and $U$ is some complementary $B$-module.

The proof is easy. Since $B$ is quasi-Frobenius, ${ }_{B} B$ is injective. Now $B^{+} \subseteq A^{+}$so that $\left.{ }_{A} A\right|_{B} \simeq{ }_{B} B \oplus W$ for some $B$-module $W$. The subalgebra $B$ is commutative. So in a complete decomposition of ${ }_{B} B$ into indecomposables, each indecomposable summand appears with multiplicity one. For some $s \mid t,{ }_{A} A \simeq s \times V$ where $V$ is the irreducible $A$-module uniquely determined up to isomorphism. Using the Krull-Schmidt theorem to compare complete decompositions of the isomorphic modules ${ }_{B} B \oplus W$ and $s \times\left. V\right|_{B}$ we discover that ${ }_{B} B$ is isomorphic to a summand of $\left.V\right|_{B}$.

(1.2) If in (1.1) the dimension of $B$ is $t$ then $\left.V\right|_{B} \simeq{ }_{B} B$.

(1.3) Suppose $\boldsymbol{k}$ is a field and $g(x) \in \boldsymbol{k}[x]$ has positive degree. Then $\boldsymbol{k}[x] /(g(x))$ is a quasi-Frobenius algebra.

This is well known. See [2, Section 58, Problem 2(c)].

\section{A nonsingular matrix.}

(2.1) Let $r$ be an odd prime and $K=\mathrm{GF}(r)$. Let $V$ be an $e \geqq 1$ dimensional vector space over $K$ and $f: V \times V \rightarrow K$ a nonsingular symmetric form.

Since $Z / r Z \simeq G F(r)$ for the integers $Z$, we may imagine that each $a \in K$ is a least residue in $Z$.

(2.2) If $\boldsymbol{k}$ is a field of characteristic $q \neq r$ containing a primitive rth root of unity $\zeta$ then the matrix $M=\left[\zeta^{\alpha \beta}\right]_{(\alpha, \beta) \in K \times K}$ is nonsingular.

Let $N=\left[\zeta^{-\alpha \beta}\right]$. Then the $(\beta, \gamma)$ entry of $M N$ is

$$
\sum_{\alpha} \zeta^{\beta \alpha-\alpha \gamma}=\sum_{\alpha} \zeta^{(\beta-\gamma) \alpha}=0
$$

unless $\beta=\gamma$ in which case it is $r$. So $M N=r I$ where $I$ is the identity. Since $r \neq 0$ in $k, M$ has inverse $r^{-1} N$.

(2.3) If $\boldsymbol{k}$ is a field of characteristic $q \neq \boldsymbol{r}$ containing a primitive rth root of unity $\zeta$ then the matrix $M=\left[\zeta^{f(u, v)}\right]_{(u, v) \in V \times V}$ is nonsingular. 
We proceed by induction on $\operatorname{dim} V$. If $\operatorname{dim} V=1$ then $f(\alpha u, \beta u)=\alpha \beta \mu$ for some $\mu \neq 0$ in $K$ so $M$ is the matrix of (2.2) with $\zeta \mu$ in place of $\zeta$ and (2.3) holds in this case.

Suppose $\operatorname{dim} V=e$ and (2.3) holds for all spaces of smaller dimension. Let $\left\{v_{1}, \cdots, v_{e}\right\}$ be an orthogonal basis for $V$. Such a basis exists since $r$ is odd. Let $U=\left\langle v_{2}, \cdots, v_{e}\right\rangle$. Now $f\left(v_{1}, v_{1}\right)=\mu$. Set $B=\left[\zeta^{\alpha \beta \mu}\right]$. Then we may arrange $M$ into blocks

$$
\begin{aligned}
M & =\left[\zeta^{f\left(\alpha v_{1}+u, \beta v_{1}+v\right)}\right] \\
& =\left[\zeta^{\alpha \beta \mu+f(u, v)}\right]=\left[B \zeta^{f(u, v)}\right] \\
& =B \otimes\left[\zeta^{f(u, v)}\right]_{(u, v) \in U \times U} .
\end{aligned}
$$

So our matrix is formed as a Kronecker product. Now $\operatorname{dim} U=e-1$ so the second matrix in the product is nonsingular. The first matrix is just the matrix of (2.2) for some primitive root; hence is nonsingular. Therefore $M$ is nonsingular.

As a corollary we obtain the following:

(2.4) Let $\tilde{\boldsymbol{K}}=\mathrm{GF}\left(r^{e}\right)$. Let $\Gamma \in \tilde{\boldsymbol{K}}^{\times}, \operatorname{Tr}: \tilde{\boldsymbol{K}} \rightarrow \boldsymbol{K}$ the trace map, $V=\tilde{\boldsymbol{K}}^{+}$, and $f(u, v)=\operatorname{Tr}(\Gamma u v)$. Let $\zeta$ be a primitive rth root in $\boldsymbol{k}$ of characteristic $q \neq r$. If $\phi_{u} \in \boldsymbol{k}, u \in \tilde{K}$ and $\sum_{u} \phi_{u} \zeta^{(f u, v)}=0$ for all $v \in \tilde{K}$, then all $\phi_{u}=0$.

The $\phi_{u}$ 's give a linear dependence on the columns of $M$ in (2.3). So this is obvious.

III. The group. Let $r$ be an odd prime and $e \geqq 1$ an integer. Let $K=$ $\mathrm{GF}(r), \tilde{\boldsymbol{K}}=\mathrm{GF}\left(r^{e}\right)$, and $\hat{\boldsymbol{K}}=\mathrm{GF}\left(r^{2 e}\right)$. Let $\mathscr{G}$ be the Galois group of $\hat{\boldsymbol{K}} / \boldsymbol{K}$ and $\phi \in \mathscr{G}$ the element of order two in $\mathscr{G}$. Let $\mu \in \hat{K}^{+}$be of order $r^{e}+1$. Set $v=\mu-\mu^{-1}$. For $u, v \in \hat{K}^{+}$set

$$
h(u, v)=2^{-1} \operatorname{Tr}\left(v\left[u v^{\phi}-u^{\phi} v\right]\right)
$$

where $\operatorname{Tr}: \tilde{\boldsymbol{K}} \rightarrow \boldsymbol{K}$ is the trace map.

Note that $v\left[u v^{\phi}-u^{\phi} v\right] \in \tilde{\boldsymbol{K}}$, the fixed field of $\phi$. Thus $h$ is a nonsingular alternating form on $\hat{\boldsymbol{K}}^{+}$. Let $R=\hat{\boldsymbol{K}}^{+} \times \boldsymbol{K}^{+}$. For $(u, \zeta),(v, \xi) \in R$ set

$$
(u, \zeta)(v, \xi)=(u+v, h(u, v)+\zeta+\xi) .
$$

This multiplication makes $R$ into an extra special $r$ group of exponent $r$ and order $r^{2 e+1}$.

Let $C=\langle\mu\rangle$. Now $C$ acts as automorphisms of $R$ by

$$
(u, \zeta)^{x}=(u x, \zeta) \text { for } x \in C, \quad(u, \zeta) \in R .
$$

We let $G=C R$ be the semidirect product of $R$ by $C$. 
If $G_{0}=C_{0} R_{0}$ where $R_{0}$ is a normal extra special $r$ subgroup of $G_{0}$ with $Z\left(R_{0}\right)=Z\left(G_{0}\right)$ and $C_{0}$ is a cyclic $r^{\prime}$ group irreducible on $R_{0} / Z\left(R_{0}\right)$ then $G_{0}$ is isomorphic to a subgroup of $G$. This fact seems to be well known. In any case it is a straightforward computation.

IV. The group algebra of $C R$. Let $\boldsymbol{k}$ be a field of characteristic $q \neq r$ which contains a primitive $r$ th root of 1 .

We now state some facts about the group algebra of $R$ over $k$. Let $\lambda$ be a primitive $r$ th root of unity in $k$. If $z=(0,1) \in R$ then set

$$
E=r^{-1}\left(\hat{\lambda}^{r-1}+\lambda^{r-2} z+\cdots+\lambda z^{r-2}+z^{r-1}\right)
$$

and

$$
\begin{aligned}
\chi((u, \zeta)) & =0 & \text { if } & u \neq 0 \\
& =r^{e} \lambda^{\zeta} & \text { if } & u=0
\end{aligned}
$$

Then $E$ is the primitive central idempotent of $k[R]$ belonging to the irreducible character $\chi$. Also $k[R] E$ is a central simple algebra of dimension $\boldsymbol{r}^{2 e}$ over $\boldsymbol{k}$.

Let $c \in C^{\#}$ and set

and

$$
\mathcal{O}_{c}=\left\{\left(v,-h\left(v v^{\phi}(c-1)^{-1}, 1\right)\right) \mid v \in \hat{\boldsymbol{K}}^{+}\right\}
$$

$$
K_{c}=-r^{-e} \sum_{x \in \mathcal{O}_{c}} x E .
$$

$$
\text { If } x \in R \text { and } c \in C^{\#} \text { then } K_{c} x^{c}=x K_{c} \text {. }
$$

Before starting we note a few properties of the form $h$. If $u, v \in \hat{K}$ then

$$
h(u, v)=h\left(v^{\phi}, u^{\phi}\right)=-h(v, u)=h\left(u v^{\phi}, 1\right)=-h\left(v u^{\phi}, 1\right) .
$$

We let $h(u)=h(u, 1)$. There should be no confusion since the two $h$ 's are related and functions of different numbers of variables. Now $h(u)$ is a nontrivial $\boldsymbol{K}$-linear functional from $\hat{\boldsymbol{K}}$ to $\boldsymbol{K}$.

For $x=(u, \delta)$, we compute

$$
\begin{aligned}
& -r^{e} x^{-1} K_{c} x^{c}=\sum_{v}(u, \delta)^{-1}\left(v,-h\left(v v^{\phi}[c-1]^{-1}\right)\right)(u, \delta)^{c} E \\
& =\sum_{v}(-u,-\delta)\left(v,-h\left(v v^{\phi}[c-1]^{-1}\right)\right)(u c, \delta) E \\
& =\sum_{t}\left(t,-h\left([t-u(c-1)][t-u(c-1)]^{\phi}(c-1)^{-1}\right.\right. \\
& \left.\left.\quad+u[t-u(c-1)]^{\phi}-[t-u(c-1)] u^{\phi} c^{-1}+u u^{\phi} c^{-1}\right)\right) E .
\end{aligned}
$$

Here we have substituted $t=v+u(c-1)$. Proceeding further,

$$
=\sum\left(t,-h\left(t t^{\phi}(c-1)^{-1}\right)\right)\left(0,-h\left(u u^{\phi}\right)\right) E .
$$


But $u u^{\phi} \in \tilde{K}$ so $h\left(u u^{\phi}\right)=0$. Thus $-r^{e} x^{-1} K_{c} x^{c}=-r^{e} K_{c}$. This proves (4.3). We now compute $K_{c} K_{d}$ for $c, d \in C^{\#}$.

$$
\begin{aligned}
r^{2 e} K_{c} K_{d} & =\sum_{u}\left(u,-h\left(u u^{\phi}(c-1)^{-1}\right)\right) \sum_{v}\left(v,-h\left(v v^{\phi}(d-1)^{-1}\right)\right) E \\
& =\sum_{u, v}\left(u+v,-h\left(u u^{\phi}(c-1)^{-1}+v v^{\phi}(d-1)^{-1}-u v^{\phi}\right)\right) E .
\end{aligned}
$$

Let $t=u+v$ and compute,

$$
\begin{aligned}
& =\sum_{t, v}\left(t,-h\left(t t^{\phi}(c-1)^{-1}+t v^{\phi}\left(c^{-1}-1\right)^{-1}-v t^{\phi}(c-1)^{-1}\right.\right. \\
& \left.\left.\quad+v v^{\phi}\left[1+(c-1)^{-1}+(d-1)^{-1}\right]\right)\right) E . \\
& \text { (4.4) For } c \in C^{\#}, K_{c} K_{c^{-1}}=E .
\end{aligned}
$$

From our computation of $K_{c} K_{d}$ we obtain,

$$
\begin{aligned}
r^{2 e} K_{c} K_{c}-1= & \sum_{t, v}\left(t,-h\left(t t^{\phi}(c-1)^{-1}+t v^{\phi}\left(c^{-1}-1\right)^{-1}-v t^{\phi}(c-1)^{-1}\right.\right. \\
& \left.\left.+v v^{\phi}\left[1+(c-1)^{-1}+\left(c^{-1}-1\right)^{-1}\right]\right)\right) E \\
= & \sum_{t}\left(t,-h\left(t t^{\phi}(c-1)^{-1}\right)\right) \\
& \cdot \sum_{v}\left(0,-h\left(t v^{\phi}\left(c^{-1}-1\right)^{-1}-v t^{\phi}(c-1)^{-1}\right)\right) E .
\end{aligned}
$$

Now

$$
t v^{\phi}\left(c^{-1}-1\right)^{-1}-v t^{\phi}(c-1)^{-1}=\left[t\left(c^{-1}-1\right)^{-1}\right] v^{\phi}-v\left[t\left(c^{-1}-1\right)^{-1}\right]^{\phi} .
$$

Set $A(t)=t\left(c^{-1}-1\right)^{-1}$. Then the second term of the above expression is

$$
\sum_{v}\left(0,-h\left(A(t) v^{\phi}-A(t)^{\phi} v\right)\right) E .
$$

But $-h\left(A(t) v^{\phi}-A(t)^{\phi} v\right)=f(v)$ is a nontrivial $\boldsymbol{K}$-linear map of $\hat{\boldsymbol{K}}^{+}$onto $\boldsymbol{K}$ provided $t \neq 0$. But then the sum is

$$
\begin{aligned}
\sum_{v}(0, f(v)) E & =r^{2 e-1} \sum_{\alpha \in K}(0, \alpha) E & & \text { if } t \neq 0, \\
& =r^{2 e}(0,0) E & & \text { if } t=0 .
\end{aligned}
$$

Now $\sum_{\alpha}(0, \alpha) E=\sum_{\alpha} \lambda^{\alpha} E=0$. So our sum is zero unless $t=0$. Then we get

$$
r^{2 e} K_{c} K_{c}-1=r^{2 e} E \text {. }
$$

We now have the obvious corollary:

(4.5) If $c \in C^{\#}$ then $K_{c}$ is invertible in $k[R] E$ and has inverse equal to $K_{c-1}$.

Let $K_{1}=E$.

(4.6) The map cx $\rightarrow K_{c} x E$ is a representation of $C R$ in $k[R] E$. 
The collection $\{(v, 0) E \mid v \in \hat{\boldsymbol{K}}\}$ is a $\boldsymbol{k}$-basis for $\boldsymbol{k}[R] E$. Also $\boldsymbol{k}[R] E$ is a central simple algebra over $\boldsymbol{k}$. Thus $c x \rightarrow K_{c} x E$ is a projective representation of $C R$ with a factor set $n$ by (4.3) and (4.5). Further, $n$ is trivial on $R$. That is, $K_{c}{ }^{-1}(v, 0) K_{c}=(v, 0)^{c}$ all $v \in \hat{\boldsymbol{K}}$.

But then $K_{c} K_{d}=n(c, d) K_{c d}$. We continue our earlier computation from (*) for the case $d \neq c^{-1}$.

$$
\begin{aligned}
r^{2 e} K_{c} K_{d}= & \sum_{t}\left(t,-h\left(t t^{\phi}(c d-1)^{-1}\right)\right) \\
& \cdot \sum_{v}\left(0,-h\left(t t^{\phi}\left[(c-1)^{-1}-(c d-1)^{-1}\right]+t v^{\phi}\left(c^{-1}-1\right)^{-1}\right.\right. \\
& \left.\left.\quad-v t^{\phi}(c-1)^{-1}+v v^{\phi}\left[1+(c-1)^{-1}+(d-1)^{-1}\right]\right)\right) E .
\end{aligned}
$$

Put the last sum equal to $A^{\prime}(t)$. Then

$$
\begin{aligned}
& =\sum_{t}\left(t,-h\left(t t^{\phi}(c d-1)^{-1}\right)\right) A^{\prime}(t) E \\
& =-r^{e} n(c, d) \sum_{t}\left(t,-h\left(t t^{\phi}(c d-1)^{-1}\right)\right) E .
\end{aligned}
$$

Since $A^{\prime}(t)$ is a sum of elements from $Z(R), A^{\prime}(t) E=a(t) E$ where $a(t) \in \boldsymbol{k}$. Further, $\left\{\left(t,-h\left(t t^{\phi}(c d-1)^{-1}\right)\right) E \mid t \in \hat{K}\right\}$ is a $\boldsymbol{k}$-basis for $\boldsymbol{k}[R] E$. Therefore $-r^{e} n(c, d)=a(t)$ for all values of $t$. In particular,

$$
\begin{aligned}
-r^{e} n(c, d) E & =a(0) E \\
& =\sum_{v}\left(0,-h\left(v v^{\phi}\left[1+(c-1)^{-1}+(d-1)^{-1}\right]\right)\right) E .
\end{aligned}
$$

The map $v \rightarrow v v^{\phi}$ is the norm map $N: \hat{\boldsymbol{K}}^{\times} \rightarrow \tilde{\boldsymbol{K}}^{\times}$. The kernel has order $r^{e}+1$. Therefore

$$
\begin{aligned}
-r^{e} n(c, d) E & =E+\sum_{v \in \hat{K}^{\times}}\left(0,-h\left(v v^{\phi}\left[1+(c-1)^{-1}+(d-1)^{-1}\right]\right)\right) E \\
& =E+\left(r^{e}+1\right) \sum_{u \in \tilde{K}^{X}}\left(0,-h\left(u\left[1+(c-1)^{-1}+(d-1)^{-1}\right]\right)\right) E \\
& =-r^{e} E+\left(r^{e}+1\right) \sum_{u \in \tilde{K}}\left(0,-h\left(u\left[1+(c-1)^{-1}+(d-1)^{-1}\right]\right)\right) E
\end{aligned}
$$

Now $-h\left(u\left[1+(c-1)^{-1}+(d-1)^{-1}\right]\right)=f(u)$ is a nontrivial $K$-linear map of $\tilde{\boldsymbol{K}}$ to $\boldsymbol{K}$ since $d \neq c^{-1}$. Thus

$$
\begin{aligned}
-r^{e} n(c, d) E & =-r^{e} E+\left(r^{e}+1\right) \sum_{u}(0, f(u)) E \\
& =-r^{e} E+r^{\rho-1}\left(r^{e}+1\right) \sum_{\alpha \in K}(0, \alpha) E=-r^{e} E .
\end{aligned}
$$


Thus $n(c, d)=1$ if $d \neq c^{-1}$. By (4.4) and the definition of $K_{1}$ we know that

$$
n(1, c)=n(c, 1)=n\left(c, c^{-1}\right)=n\left(c^{-1}, c\right)=1 .
$$

So $n$ is the trivial factor set and we have an ordinary representation.

$$
\sum_{c \in C^{\#}} K_{c}=-E
$$

As usual, we compute

$$
\begin{aligned}
-r^{e} \sum_{c \in C^{\#}} K_{c} & =\sum_{c, v}\left(v,-h\left(v v^{\phi}(c-1)^{-1}\right)\right) E \\
& =\sum_{v}(v, 0) \sum_{c}\left(0,-h\left(v v^{\phi}(c-1)^{-1}\right)\right) E .
\end{aligned}
$$

Fix $v \neq 0$. Compute

$$
\begin{aligned}
-h\left(v v^{\phi}(c-1)^{-1}\right) & =-\operatorname{Tr}\left(2^{-1} v v v^{\phi}\left[(c-1)^{-1}-\left(c^{-1}-1\right)^{-1}\right]\right) \\
& =-\operatorname{Tr}\left(2^{-1} v v^{\phi} v(c+1) /(c-1)\right) .
\end{aligned}
$$

Next we show that the map $c \rightarrow v(c+1) /(c-1)$ is a one-one map of $C^{\#}$ onto $\tilde{\boldsymbol{K}}$. Note that $(v(c+1) /(c-1))^{\phi}=v(c+1) /(c-1)$ so the map is into $\tilde{\boldsymbol{K}}$. Since $\left|C^{\#}\right|=r^{e}=|\tilde{\boldsymbol{K}}|$, if the map is one-one, it is onto. So suppose

$$
v \frac{c+1}{c-1}=v \frac{d+1}{d-1}, \quad c, d \in C^{\#} .
$$

Then $(c+1)(d-1)=(d+1)(c-1)$ or $2(c-d)=0$. But $r$ is odd so $c=d$. Thus the map is one-one onto $\tilde{\boldsymbol{K}}$.

Returning again to our computation

$$
\begin{aligned}
-r^{e} \sum_{c \in C^{\#}} K_{c} & =\sum_{v}(v, 0) \sum_{u \in \tilde{K}}\left(0,-\operatorname{Tr}\left(2^{-1} v v^{\phi} u\right)\right) E \\
& =r^{e}(0,0) E+\sum_{v \neq 0}(v, 0) r^{e-1} \sum_{\alpha \in K}(0, \alpha) E=r^{e} E .
\end{aligned}
$$

This proves (4.7).

(4.8) $\left\{K_{c} \mid c \in C^{\#}\right\}$ is a linearly independent set of vectors in $k[R] E$.

Suppose there are constants $\psi_{c} \in \boldsymbol{k}$ so that $\sum_{c \in C^{\#}} \psi_{c} K_{c}=0$. Then

$$
\begin{aligned}
0 & =-r^{e} \sum_{c \in C^{\#}} \psi_{c} K_{c}=\sum_{c \in C^{\#} ; v \in \hat{K}} \psi_{c}\left(v,-h\left(v v^{\phi}(c-1)^{-1}\right)\right) E \\
& =\sum_{v \in \hat{K}}(v, 0) \sum_{c} \psi_{c}\left(0,-h\left(v v^{\phi}(c-1)^{-1}\right)\right) E .
\end{aligned}
$$


But $\{(v, 0) E \mid v \in \hat{\boldsymbol{K}}\}$ is a $\boldsymbol{k}$-basis for $\boldsymbol{k}[R] E$. Therefore

$$
\sum_{c} \psi_{c}\left(0,-h\left(v v^{\phi}(c-1)^{-1}\right)\right) E=0
$$

for all $v \in \hat{K}$. But $v \rightarrow v v^{\phi}$ is the norm map from $\hat{K}$ to $\tilde{K}$ and is onto. So

for all $\alpha \in \tilde{\boldsymbol{K}}$.

$$
\sum_{c} \psi_{c}\left(0,-h\left(\alpha(c-1)^{-1}\right)\right) E=0
$$

Next look at

$$
\begin{aligned}
h\left(\alpha(c-1)^{-1}\right) & =2^{-1} \operatorname{Tr}\left(\nu \alpha\left[(c-1)^{-1}-\left(c^{-1}-1\right)^{-1}\right]\right) \\
& =2^{-1} \operatorname{Tr}(\alpha \nu(c+1) /(c-1)) .
\end{aligned}
$$

In (4.7) we saw that the map $c \rightarrow v(c+1) /(c-1)$ was one-one from $C^{\#}$ onto $\tilde{\boldsymbol{K}}$. Let $\beta=\beta(c)=v(c+1) /(c-1)$. Then we may take $\psi_{c}=\psi_{\beta(c)}^{\prime}=\psi_{\beta}^{\prime}$ and

$$
0=\sum_{\beta \in \tilde{K}} \psi_{\beta}^{\prime}(0, f(\alpha, \beta)) E
$$

for all $\alpha \in \tilde{K}$ where $f(\alpha, \beta)=-2^{-1} \operatorname{Tr}(\alpha \beta)$ is a nonsingular symmetric form from $\tilde{\boldsymbol{K}} \times \tilde{\boldsymbol{K}}$ to $\boldsymbol{K}$. That is,

$$
0=\sum_{\beta \in \tilde{K}} \psi_{\beta}^{\prime} \lambda^{f(\alpha, \beta)} \quad \text { for all } \alpha \in \tilde{K}
$$

By (2.4) all $\psi_{\beta}^{\prime}=0$. This proves (4.8).

(4.9) Define $\Phi(c)=K_{c}$ and extend linearly to $k[C]$. Then $\Phi$ is an algebra homomorphism with kernel $k[C] F$ where $F=\sum_{x \in C} x$.

This is an easy consequence of (4.6), (4.7), and (4.8).

(4.10) THEOREM. Let $V$ be an irreducible $k[C R]$-module nontrivial on $\boldsymbol{Z}(R)$. Then there is a $\boldsymbol{k}[C]$-module $W$ so that

$$
\left.V\right|_{C} \simeq(k[C] / k[C] F) \otimes_{k} W .
$$

Let $E$ be the primitive central idempotent of $k[R]$ such that $E V \neq(0)$. For appropriate choice of $\lambda$ in $(4.1), E$ is given there. Let $B$ be the $k$ subalgebra generated by the $K_{c}$ 's. Let $U$ be an irreducible $k[R]$ module with $E U \neq(0)$. Then $U$ is a $k[C R]$ module if we let $c \in C$ act as $K_{c}$. By (1.2), (1.3) and (4.9), $\left.U\right|_{C} \simeq{ }_{B} B$.

So by $[2,(51.7)]$ there is a $C R / R \simeq C$-module $W$ such that

$$
V \simeq U \otimes_{k} W \text { or }\left.\left.V\right|_{C} \simeq(\boldsymbol{k}[C] / k[C] F) \otimes W\right|_{C}
$$

We have the following easy corollary. 
(4.11) CoRollary. Let $D$ be a subgroup of $C$ and $V$ an irreducible $\boldsymbol{k}[C R]$-module faithful on $Z(R)$. Then there is a $\boldsymbol{k}[D]$-module $W$ so that

$$
\left.V\right|_{D} \simeq(\operatorname{dim} W)\left(\left(r^{e}+1\right) /|D|-1\right) k[D] \oplus\left(\boldsymbol{k}[D] / \boldsymbol{k}[D] F_{0}\right) \otimes W
$$

where $F_{0}=\sum_{x \in D} x$.

We have completed the proof of the theorem. This construction need not be sonfined to $\boldsymbol{k}$. With modification it gives a representation of $C R$ in $\mathcal{O}$, the ring of $p$-adic integers $p \neq r$, in $k$ when $k$ is an algebraic number field.

Is there a characteristic free proof for $r=2$ ?

\section{REFERENCES}

1. T. R. Berger, Class two p groups as fixed point free automorphism groups, Illinois J. Math. 14 (1970), 121-149. MR 41 \#336.

2. C. W. Curtis and I. Reiner, Representation theory of finite groups and associative algebras, Pure and Appl. Math., vol. 11, Interscience, New York, 1962. MR 26 \#2519.

3. P. Hall and G. Higman, On the p-length of p-soluble groups and reduction theorems for Burnside's problem, Proc. London Math. Soc. (3)6(1956), 1-42. MR 17, 344.

4. J. G. Thompson, Vertices and sources, J. Algebra 6 (1967), 1-6. MR 34 \#7677.

5. E. E. Shult, On groups admitting fixed point free abelian operator groups, Illinois J. Math. 9 (1965), 701-720. MR 32 \#1269.

Department of Mathematics, University of Minnesota, Minneapolis, MinNESOTA 55455 